\title{
A synchrotron radiation diagnostic to observe relativistic runaway electrons in a tokamak plasma
}

\author{
R. Jaspers, ${ }^{\text {a) }}$ N. J. Lopes Cardozo, A. J. H. Donné, and H. L. M. Widdershoven \\ FOM Instituut voor Plasmafysica 'Rijnhuizen', Association EURATOM-FOM, \\ Partner in the Trilateral Euregio Cluster, Postbus 1207, 3430 BE Nieuwegein, The Netherlands \\ K. H. Finken \\ Institut für Plasmaphysik, Association EURATOM-KFA, Partner in the Trilateral Euregio Cluster, \\ Forschungszentrum Jülich GmbH, D-52425 Jülich, Germany
}

(Presented on 19 June 2000)

In present day tokamaks runaway electrons can be confined long enough to gain energies in the order of several tens of megaelectron volts. At these energies synchrotron radiation is emitted in the infrared wavelength range which can easily be detected by thermographic cameras. The spectral features of this synchrotron radiation are reviewed. On TEXTOR-94 a diagnostic exploiting this synchrotron radiation has been developed and is presented here. It is shown how to deduce the runaway parameters like runaway energy, pitch angle, runaway current and beam radius from the measurements. Based on the experience at TEXTOR-94 the feasibility of a similar synchrotron diagnostic on the International Thermonuclear Experimental Reactor is discussed. The maximum emission is expected in the wavelength range from 1-5 $\mu \mathrm{m}$. A beam of $10 \mathrm{MeV}$ runaway electrons with a current of about $15 \mathrm{kA}$ will already be detectable. (C) 2001 American Institute of Physics.

[DOI: $10.1063 / 1.1318245$ ]

\section{INTRODUCTION}

Several tokamaks have reported on the observation of runaway electrons of several tens of megaelectron volts, generated mainly in disruptive events. ${ }^{1,2}$ Also in steady state, low-density discharges, runaway electrons can be confined long enough to gain energies of this magnitude. ${ }^{3}$ At these energies synchrotron radiation is emitted in the (near) infrared wavelength range which can easily be detected by commercially available thermographic cameras. This provides an easy technique for in situ runaway electron studies.

Runaway electrons are being investigated for several reasons. For future fusion reactors, it is of major importance to know the processes of runaway generation and runaway loss after disruptions, because of the severe damage the local loss of large amounts of these highly energetic electrons may cause on first wall components. ${ }^{4}$ Second, since the runaway electrons are effectively collisionless, their confinement is determined by the magnetic field turbulence. In this way the runaway transport provides a unique opportunity to probe turbulence in the core of a thermonuclear plasma. ${ }^{5}$

For these studies the synchrotron radiation is the most powerful tool to diagnose the relativistic runaway electron distribution. This diagnostic provides a direct image of the runaway beam inside the plasma. From the spectral features the runaway energy can in principle be obtained, the intensity of the radiation is a measure of the number of runaway electrons, and the shape of the synchrotron spot exhibits information about their perpendicular momentum.

At the medium sized circular limiter tokamak TEXTOR-94 (major radius $R_{0}=1.75 \mathrm{~m}$, minor radius $a$

${ }^{a)}$ Electronic mail: r.jaspers@fz-juelich.de
$=0.46 \mathrm{~m}$ ) runaway electrons are studied with a thermographic camera detecting this synchrotron radiation. ${ }^{3}$ This camera operates in the wavelength range of $3-8 \mu \mathrm{m}$ and has a full poloidal view. Runaway electrons with energies of 25-30 MeV have been observed in low-density steady state discharges ${ }^{6}$ as well as during disruptions. ${ }^{7}$ Information about runaway generation mechanisms, runaway snakes, runaway losses in stochastic fields and scale size of magnetic turbulence could be deduced using this synchrotron radiation diagnostic. ${ }^{5,6,8}$

After a short introduction into the characteristics of the synchrotron radiation in tokamaks in Sec. II, the diagnostic as presently operational at TEXTOR-94 will be described in Sec. III. It will be shown how to deduce the runaway electron parameters, such as energy, pitch angle, number, and beam radius from synchrotron radiation measurements. This is done in Sec. IV.

Based on the experience at TEXTOR-94 the feasibility of a similar synchrotron diagnostic on the International Thermonuclear Experimental Reactor (ITER) is discussed in Sec. $\mathrm{V}$. The maximum emission is expected in the wavelength range from $1-5 \mu \mathrm{m}$. The minimum detectable runaway current is calculated. The steep short wavelength slope of the synchrotron emission makes it possible to determine the maximum runaway energy by a measurement at only two different wavelengths. The compatibility with an already planned infrared (IR) wide angle viewing system is investigated.

\section{SYNCHROTRON RADIATION}

The spectral power density $P(\lambda)$ of the synchrotron radiation emitted by relativistic electrons moving on a circular orbit with radius $R$ is expressed by 


$$
\begin{aligned}
P(\lambda) & =\frac{4 \pi}{\sqrt{3}} \frac{m_{e} c^{3}}{\Gamma^{2} \lambda^{3}} \int_{w}^{\infty} K_{5 / 3}(x) d x \\
& \approx \frac{4 \pi m_{e} c^{3} r_{e}}{\Gamma^{2} \lambda^{3}} \sqrt{\frac{\pi}{2 w}} \exp (-w),
\end{aligned}
$$

where $w=4 \pi R /\left(3 \Gamma^{3} \lambda\right)$ and the last approximation is only valid for $w \gg 1$. Here $m_{e}$ is the electron rest mass, $c$ the velocity of light, $r_{e}$ the classical electron radius, $\Gamma$ the relativistic mass factor and $\lambda$ the wavelength. The emission is mainly in the forward direction with an opening angle $\delta$ of $\delta=1 / \Gamma$.

For the specific situation of runaway electrons in a tokamak, Eq. (1) is still valid when the instantaneous radius of curvature of the orbit $R_{\text {curv }}$ is approximated given by ${ }^{9}$

$$
R_{\text {curv }}^{-2} \approx R^{-2}\left[1+\eta^{2}+2 \eta \sin (\Theta+\alpha)\right]
$$

which may oscillate strongly during the motion in a tokamak. Here $v_{\|} \gg v_{\perp}, \omega_{B o}=e B_{T} / m_{e}$ and $\eta=v_{\perp} / v_{\mathrm{dr}}$, $v_{\mathrm{dr}}=\Gamma v_{\|}^{2} / \omega_{B o} R$, with $v_{\mathrm{dr}}$ the drift velocity, $\Theta$ the poloidal angle corresponding to the position of the guiding center and $\alpha$ the phase of the cyclotron gyration.

Using the Schwinger approach ${ }^{10}$ and taking into account the features of the relativistic electron motion in a tokamak (motion along the magnetic field line, cyclotron gyration, and vertical centrifugal drift) the spectral density of the emitted power was derived in Ref. 9. For the parameter $\xi=4 \pi / 3 R / \lambda \Gamma^{3} 1 / \sqrt{1+\eta^{2}} \gg 1$ it is given by $(\Gamma \gg 1)$

$$
\begin{aligned}
P(\lambda) \approx & \pi m_{e} c^{3} r_{e} \sqrt{\frac{2 \sqrt{1+\eta^{2}}}{\lambda^{5} R \Gamma}} \\
& \times\left[I_{0}(a)+\frac{4 \eta}{1+\eta^{2}} I_{1}(a)\right] \exp (-\xi),
\end{aligned}
$$

where $I_{0,1}(a)$ is the modified Bessel function and $a$ $=(4 \pi / 3)\left(R / \lambda \Gamma^{3}\right)\left(\eta /\left(1+\eta^{2}\right)^{3 / 2}\right)$. At

$$
\lambda_{m} \approx \frac{8 \pi}{15} \frac{R}{\sqrt{1+\eta^{2}}} \frac{1}{\Gamma^{3}}, \quad\left(a\left(\lambda_{m}\right) \leqslant 1.25\right)
$$

the $P(\lambda)$ expression takes a maximum value

$$
P\left(\lambda_{m}\right) \approx 0.1 m_{e} c^{3} r_{e}\left(1+\eta^{2}\right)^{3 / 2} \Gamma^{7} / R^{3} .
$$

The spectrum is shifted to smaller wavelengths compared to the case $\eta=0$. For the case $\lambda>\lambda_{m}$, when $\xi \sim 1$ it is necessary to use the general expression (15) from Ref. 9. In the case $\eta^{2} \gg 1$ the Schwinger equation [Eq. (1)] results with $R_{\text {curv }}$ $\approx \Gamma v_{\|}^{2} / v_{\perp} \omega_{B 0}$.

Due to the gyration motion of the runaway electron, the effective opening angle of the synchrotron radiation is increased and radiated in a cone with a half opening angle $\theta$ given by the pitch angle, the ratio of the perpendicular to parallel velocity: $\theta=v_{\perp} / v_{\|}$. For typical TEXTOR-94 parameters, the synchrotron spectra according to Eq. (3) are shown in Fig. 1. There, a strong dependence on energy, but also on pitch angle $\theta$, is observed. The maximum emission is in the infrared range around $5 \mu \mathrm{m}$.

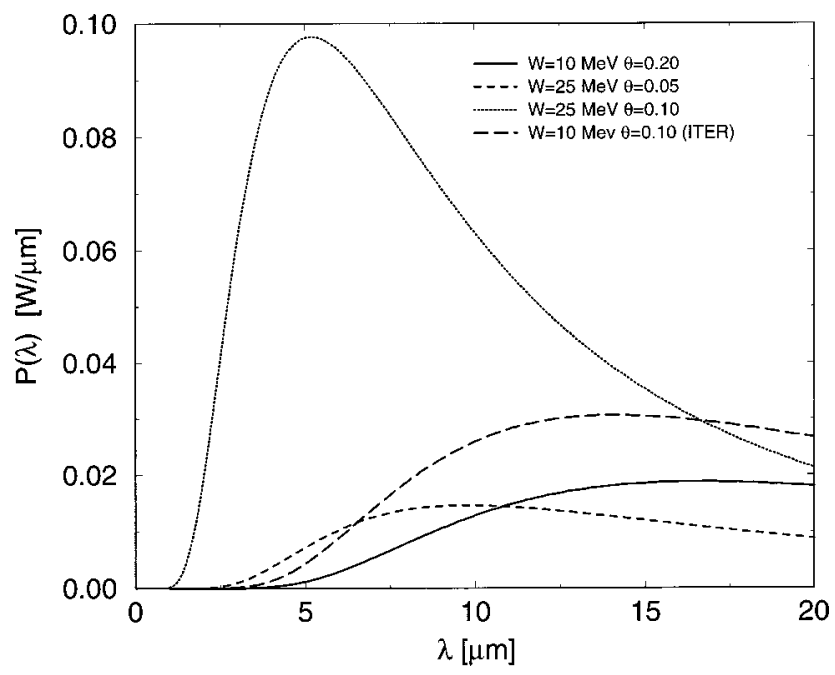

FIG. 1. Synchrotron spectra calculated for one electron for TEXTOR-94 and ITER for different values of energy $W$ and pitch angle $\theta$.

\section{TEXTOR-94 SETUP}

Synchrotron radiation measurements are routinely made at TEXTOR-94 with a thermographic camera operating in the wavelength range 3-12 $\mu \mathrm{m}$. However, since $\mathrm{CaF}_{2}$ optics are used, the effective long wavelength limit is reduced to 8 $\mu \mathrm{m}$. The thermographic camera is of the type Inframetrics $760 \mathrm{BB}$. This IR scanner consists of a HgCdTe detector cooled with liquid nitrogen. Two scanning mirrors in front of the detector scan the two-dimensional (2D) field of view in $1 / 50 \mathrm{~s}$ in the horizontal and veritcal direction, respectively. An option exists to fix the vertical mirror, resulting in a $1 \mathrm{D}$ scan at a rate of $8 \mathrm{kHz}$. The analog data was previously recorded on videotape and analyzed afterwards. Recently, the electronics have been upgraded and all signals are digitized to 12 bits before they are transferred by fiber optics to the remote computer for data analysis and storage. ${ }^{11}$ Moreover, to avoid electric or magnetic interference (especially during a disruption, when the generation of runaway electrons preserves special attention), the camera head and the electronic box are both well shielded by an iron housing with an internal copper foil.

The camera is located in the equatorial plane looking tangentially into the direction of runaway electron approach. The optical system consists of a curved mirror inside the vacuum vessel, and outside the vacuum of a $\mathrm{CaF}_{2}$ object lens, a plane mirror, a $\mathrm{CaF}_{2}$ field lens, and the IR camera including some optics and oscillating mirrors. The field of view is such that a full poloidal view of the TEXTOR-94 vessel can be seen. When no synchrotron radiation is present, the thermal radiation of the vessel wall is clearly recognized. This image, including all the wall components and diagnostic ports, provides an ideal possibility for aligning the optics and performing a spatial calibration [Fig. 2(a)].

For the synchrotron radiation, in principle, a lineintegrated signal is observed but the small opening angle of the runaway electrons limits the toroidal extension of the observed volume. For TEXTOR-94 this has been estimated to be less than $2 R_{0} \theta \approx 35 \mathrm{~cm}$ in the toroidal direction. The 

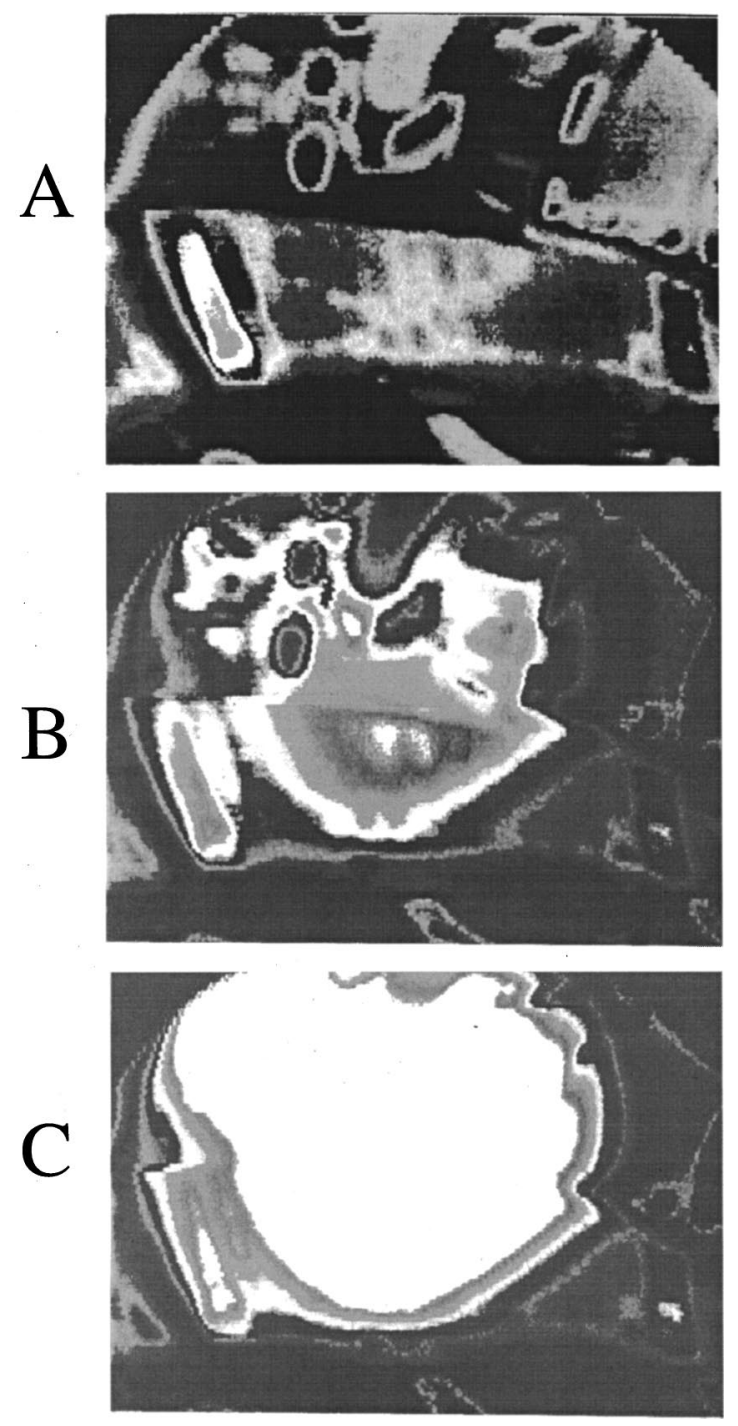

FIG. 2. (a), (b), and (c) Tangential view into a low density TEXTOR-94 discharge with a thermographic camera looking into the direction of electron approach. In frame A recorded at $t=0.5 \mathrm{~s}$ no synchrotron radiation is observable and only the wall structure can be recognized. In frame B, recorded at $t=1.5 \mathrm{~s}$, the synchrotron radiation starts to develop, and in frame $\mathrm{C}$ the full extent of the spot is visible from which the size of the runaway beam can be determined.

observed spot is not an exact poloidal cross section through the runaway beam but originates from a curved plane, due to the curvature of the runaway motion. Nevertheless, the synchrotron spot can be interpreted as a poloidal projection of the runaway beam, apart from some minor deformation as shortly discussed in the next section. Typical examples of synchrotron images is shown in Figs. 2(b) and 2(c).

\section{DEDUCTION OF RUNAWAY PARAMETERS}

As follows from Eq. (1) the synchrotron spectrum depends on two quantities representing the electron momentum: its energy $W_{r}=\Gamma m_{\theta} c^{2}$ and its radius of curvature, which is mainly determined by the pitch angle $\theta$. Since the latter parameter can be determined from the extension of the synchrotron spot (as will be shown), the energy can be, in principle, deduced from the spectrum. However, the actual situation is somewhat more complicated since a distribution in energy and pitch angle is anticipated and additional information or modeling is necessary to obtain these distributions. Nevertheless, a first indication of $\Gamma$ and $\theta$ can be obtained by assuming that the observed radiation is dominated by the highest energetic runaway electrons with the largest pitch angles, since the synchrotron radiation roughly scales like $(\Gamma \theta)^{2}$. How to deduce the runaway parameters will be briefly explained here.

\section{A. Pitch angle and beam radius}

In case the runaway electrons would only have parallel energy, only a small strip of radiation would be visible by a tangential camera. This would be a horizontal strip in case no poloidal magnetic field would be present. But this field is, of course, present and makes the field lines helical. This results in a narrow pattern, which makes an angle of approximately $\beta \approx r / R_{0} q$ ( $q$ being the safety factor) with the equatorial plane. ${ }^{12}$ The other extreme would be a large opening angle $\theta$, corresponding to a large perpendicular velocity. In that case all runaway electrons in the beam would be visible and an almost circular spot would be observed. However, the horizontal extension would be larger by the amount $d r=R(1$ $-\cos \theta) \approx R \theta^{2}$ due to the toroidal curvature of the electron orbit. An exact analysis of the shapes of the synchrotron spots including the intermediate cases is made in Ref. 12 .

TEXTOR-94 cases show that the observed spot is almost circular. This automatically indicates that the pitch angle must be sizeable, and with reasonable accuracy the vertical extent of the synchrotron spot corresponds to the beam radius and the horizontal extent is larger by the amount $R \theta^{2}$. Typical TEXTOR parameters $r_{\text {beam }}=0.25 \mathrm{~m}$ and $\theta=0.12 \mathrm{rad}$ could be determined that way.

\section{B. Runaway energy}

The runaway energy can be deduced in two different ways. First of all by exploiting the strong energy dependence of the spectrum. Measuring the synchrotron spectrum with two different interference filters in front of the IR camera allows us to quite accurately determine the runaway energy from the ratio of the measured intensities (assuming the pitch angle is known). The second method is based on the orbit shift effect of high energetic electrons. Due to the curvature drift the drift orbits of runaway electrons are shifted with respect to the magnetic surfaces. This shift $\Delta$ is given by

$$
\Delta \approx q W_{r} /\left(e c B_{\varphi}\right) .
$$

The center of the runaway beam is thus shifted with respect to the plasma center and $W_{r}$ can be calculated. Note that this method is independent of the knowledge of the pitch angle $\theta$. For TEXTOR a typical runaway energy of $25 \pm 5 \mathrm{MeV}$ has been calculated.

\section{Runaway current}

The number of runaways $N_{r}$ deduced from the synchrotron radiation is calculated from

$$
N_{r} \int P(\lambda) T(\lambda) d \lambda=\int L_{\lambda}^{s} T(\lambda) d \lambda A_{r} \Omega,
$$


where $A_{r}$ is the cross-sectional area of the ring filled with runaway electrons, $\Omega \approx \pi / \Gamma$ is the solid angle into which the radiation is emitted, $L_{\lambda}^{s}$ is the measured spectral radiance and $T(\lambda)$ is the transmission factor of the optical system. The absolute value of the radiance is obtained by comparing the synchrotron radiation with the thermal radiation from the toroidal limiter, of which the temperature is measured independently (about $650 \mathrm{~K}$ ) and of which the emissivity is known (graphite, emission coefficient $\approx 0.8$ ). While the absolute intensity can be determined rather accurately, the absolute number of runaways in the discharge can only be determined within an order of magnitude due to the uncertainty about the energy distribution. For a typical low density $\left(n_{e}(0)=1 \times 10^{19} \mathrm{~m}^{-3}, I_{p}=350 \mathrm{kA}\right)$ ohmic discharge, the runaway current is between 1 and $10 \mathrm{kA}$ if an exponentially decreasing energy distribution is assumed with $f(W)$ $\sim \exp \left(-W / W_{0}\right),\left(W_{0}=20 \mathrm{MeV}\right.$ as expected from the process of secondary generation), ${ }^{4,6}$

\section{ITER}

The issue of runaway damage in large tokamaks due to disruptions in, e.g., ITER urges for a suitable runaway diagnostic. To address the feasibility of a synchrotron radiation diagnostic for such a machine the following questions are answered in this section:

(a) What is the optimum wavelength range?

(b) What is the minimum detectable runaway current?

(c) Can runaway current and energy be monitored real time?

(d) What kind of optics system should be used?

The wavelength of maximum emission is simply calculated from Eq. (4) using the parameters $R_{0}=6.2 \mathrm{~m}, B_{t}$ $=5.3 \mathrm{~T}$. The resulting $\lambda_{\max }$ as a function of runaway energy is plotted in Fig. 3 for a pitch angle $\theta=0.10 \mathrm{rad}$. Note that an angle $\theta$ around 0.1 is expected according to the model calculations of Ref. 13 and a similar value is found for TEXTOR-94 ${ }^{3}$ and the Joint European Torus (JET). ${ }^{1}$ If the energies do not exceed $50 \mathrm{MeV}$ the infrared range between 1 and $5 \mu \mathrm{m}$ seems most appropriate for these measurements.

However, for the runaway electrons to be detectable their radiation should be distinguished from the background radiation consisting of the thermal radiation of the wall components and the plasma bremsstrahlung. For the thermal radiation Planck's law can be applied

$$
B_{\mathrm{bb}}(T)=\frac{2 h c^{2}}{\lambda^{5}}\left[\exp \left(\frac{h c}{\lambda k T}\right)-1\right]^{-1} .
$$

For the bremsstrahlung is taken

$$
\begin{aligned}
B_{\text {brem }} & =\epsilon_{\lambda} L \\
& =1.5 \times 10^{-45} \frac{n_{e}^{2} Z_{\mathrm{eff}} \bar{g}_{f f}}{\lambda^{2} \sqrt{T_{e}}} e^{-h c / \lambda T_{e}} \quad\left(\mathrm{~W} \mathrm{~m}^{-3} \mathrm{sr}^{-1} \mathrm{~m}\right)
\end{aligned}
$$

with $\epsilon_{\lambda}$ the emissivity, $g_{f f}$ the gaunt factor, and $L$ the integration length $(5 \mathrm{~m})$. Since runaway production in ITER will mainly occur during disruptions we take as values $Z_{\text {eff }}=4$,

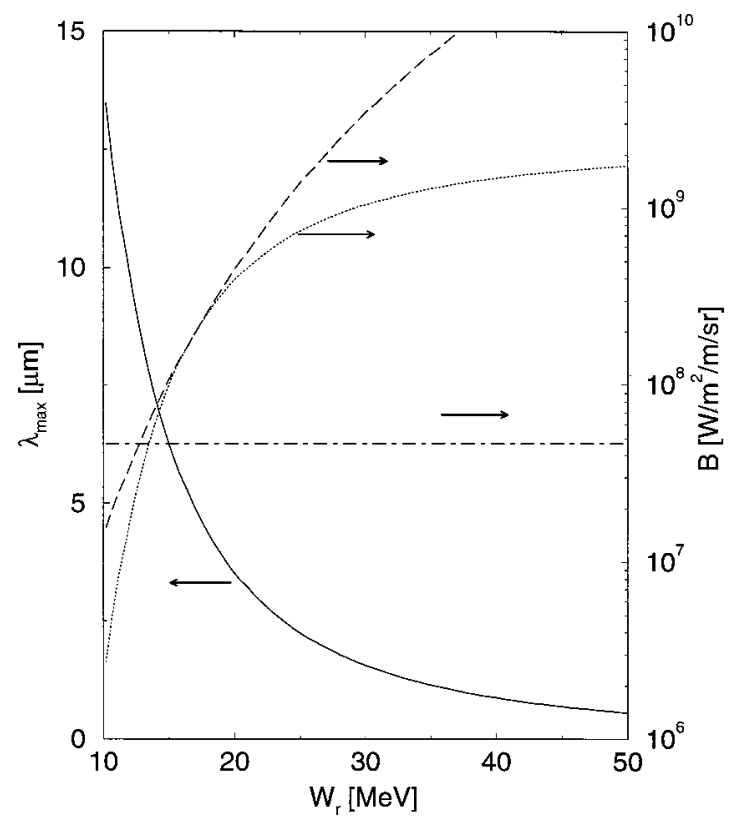

FIG. 3. The full line represents the wavelength $\lambda_{\max }$ of maximum synchrotron emission as a function of energy for ITER. It is seen that the dominant emission is in the infrared range $[1-5 \mu \mathrm{m}]$ for the energy range 20-50 $\mathrm{MeV}$. The other lines represent the synchrotron radiance $B_{\text {syn }}$ for $\lambda=5 \mu \mathrm{m}$ (dotted line) and $\lambda=\lambda_{\max }$ (dashed line). The horizontal line is the background radiance $B_{\mathrm{bb}}+B_{\mathrm{brem}}$. All calculations are done assuming a runaway current of $1 \mathrm{kA}$ and $\theta=0.10$.

$n_{e}\left[\mathrm{~m}^{-3}\right]=2 \times 10^{20}, T_{e}[\mathrm{eV}]=100$, and $h c / \lambda$ is expressed in electron volts. Inserting these values it turns out that for $\lambda<4.5 \mu \mathrm{m}$ the bremsstrahlung dominates the background and for a longer wavelength the thermal radiation.

For the synchrotron radiation is used

$$
B_{\mathrm{syn}}=\frac{2 \pi R_{0} I_{r}}{e c} \frac{P\left(\lambda_{\max }\right)}{\Omega_{r} A_{r}} .
$$

Here the first term is the runaway number, $I_{r}$ the runaway current, $\Omega_{r}=\pi / \Gamma$ the solid angle into which the synchrotron radiation is emitted, and $A_{r}$ the poloidal cross section of the runaway beam, assumed to be half of the plasma cross section. The result as a function of runaway energy is depicted in Fig. 3 for $\lambda=5 \mu \mathrm{m}$, taking typical ITER parameters $\left(A_{r}\right.$ $=11 \mathrm{~m}^{2}, T($ wall $\left.)=400 \mathrm{~K}\right)$ and assuming a runaway current $I_{r}=1 \mathrm{kA}$. It is found that for energies larger than $15 \mathrm{MeV}$ this current can be detected. At $10 \mathrm{MeV}$ and a runaway current $I_{r}=15 \mathrm{kA}$, the synchrotron intensity is half of the background and detection is possible. For even lower energies no detection will be possible at $\lambda=5 \mu \mathrm{m}$, due to the very steep short wavelength slope of the runaway electrons.

The maximum energy of the runaway beam is easily obtained from a spectral measurement. Since the short wavelength slope is steep, it is very sensitive dependent on the runaway energy. This allows us to determine (within some approximations) the runaway energy from the intensity ratio $P_{1} / P_{2}$ of the synchrotron radiation measured at two different wavelengths $\lambda_{1}$ and $\lambda_{2}$ both smaller than $\lambda_{\max }$. For $\lambda_{1,2}=3,5 \mu \mathrm{m}$ the ratio $P_{1} / P_{2}$ is almost linearly dependent on the runaway energy in the energy range $10-40 \mathrm{MeV}$. This opens the possibility for an ITER synchrotron radiation di- 
agnostic to have real time information on the runaway energy and knowing this the absolute intensity of either $P_{1}$ or $P_{2}$ provides then the real time number of runaway electrons.

What an ITER synchrotron radiation diagnostic will look like is not yet fully investigated, but the requirements (i) wide angle system for a full view of the runaway beam and (ii) in the infrared wavelength range around 1-5 $\mu \mathrm{m}$, makes it tempting to use the already planned IR wide angle system for a real time temperature monitor of the first wall. ${ }^{14}$ Concerning time resolution $(10-100 \mathrm{~ms})$, spatial resolution (5 $\mathrm{mm})$ and accuracy $\left(\Delta t=20^{\circ} \mathrm{C}\right)$ this seems suitable. Only a provision to make a spectral measurement as well should be implemented to have a monitor of the runaway energy and number.

\section{DISCUSSION}

It has been shown that the synchrotron radiation diagnostic provides a powerful tool for monitoring and investigating the runaway electrons in tokamak plasma. Commercially available thermographic cameras are well suited for detecting the synchrotron radiation. The image directly gives evidence for the presence, location, and size of the runaway beam. By carefully analyzing the spot of the picture, the intensity, and the time history additional runaway information is obtained. However, for a precise evaluation of runaway energy and pitch angle, and eventually its distribution, a spectral measurement would make the interpretation easier. Moreover, for a consistent interpretation of the runaway electron behavior, measurements at lower energies are required. The possibilities here include nonthermal electron cyclotron radiation, neutrons, or hard $\mathrm{x}$ rays. Although present day advances in diagnostic development open the possibility for some $2 \mathrm{D}$ imaging cameras in these wavelength ranges, the runaway information obtained is only indirect and will be more difficult to interpret.

\section{ACKNOWLEDGMENTS}

The authors would like to express their gratitude to Dr. Pankratov, Dr. T. Denner, and Dr. I. Entrop for helpful information and material used in this article. The work described in this article was performed as part of the research program of the "Stichting voor Fundamenteel Onderzoek der Materie (FOM)", with financial support from the "Nederlandse Organisatie voor Wetenschappelijk Onderzoek (NWO)', and EURATOM.

${ }^{1}$ R. D. Gill, B. Alper, A. W. Edwards, L. C. Ingesson, M. F. Johnson, and D. J. Ward, Nucl. Fusion 40, 163 (2000).

${ }^{2}$ R. Yoshino, T. Kondoh, Y. Neyatani, K. Itami, Y. Kawano, and N. Isei, Plasma Phys. Controlled Fusion 39, 313 (1997).

${ }^{3}$ K. H. Finken, J. G. Watkins, D. Rusbüldt, W. J. Corbett, K. H. Dippel, D. M. Goebel, and R. A. Moyer, Nucl. Fusion 30, 859 (1990).

${ }^{4}$ S. Putvinski, P. Barabaschi, N. Fujisawa, N. Putvinskaya, M. N. Rosenbluth, and J. Wesley, Plasma Phys. Controlled Fusion 39, B157 (1997).

${ }^{5}$ I. Entrop, N. J. Lopes Cardozo, R. Jaspers, and K. H. Finken, Phys. Rev. Lett. 84, 3606 (2000).

${ }^{6}$ R. Jaspers, K. H. Finken, G. Mank, F. Hoenen, J. Boedo, N. J. Lopes Cardozo, and F. C. Schüller, Nucl. Fusion 33, 1775 (1993).

${ }^{7}$ R. Jaspers, N. J. Lopes Cardozo, F. C. Schüller, K. H. Finken, T. Grewe, and G. Mank, Nucl. Fusion 36, 367 (1996).

${ }^{8}$ I. Entrop, R. Jaspers, N. J. Lopes Cardozo, and K. H. Finken, Plasma Phys. Controlled Fusion 41, 377 (1999); R. Jaspers, N. J. Lopes Cardozo, K. H. Finken, B. C. Schokker, G. Mank, G. Fuchs, and F. C. Schüller, Phys. Rev. Lett. 72, 4093 (1994).

${ }^{9}$ I. M Pankratov, Plasma Phys. Rep. 25, 145 (1999).

${ }^{10}$ J. Schwinger, Phys. Rev. 75, 1912 (1949).

${ }^{11}$ K. H. Finken, T. Denner, and G. Mank, Nucl. Fusion 40, 339 (2000).

${ }^{12}$ I. M. Pankratov, Plasma Phys. Rep. 22, 535 (1996).

${ }^{13}$ M. N. Rosenbluth and S. V. Putvinski, Nucl. Fusion 37, 1335 (1997).

${ }^{14} \mathrm{G}$. Vayakis, ITER (private communications). 